\title{
Effect of Low-Level Laser Therapy in Treatment of Chemotherapy Induced Oral Mucositis
}

\author{
Soheir Shehata Rezk-Allah' ${ }^{*}$, Heba Mohamed Abd Elshafi', Reem Jan Farid², Mohamed Abd Elrahman \\ Hassan $^{3}$, Samy A. Alsirafy ${ }^{4}$ \\ ${ }^{1}$ Basic Science Department, Faculty of Physical Therapy, Cairo University, Cairo, Egypt \\ ${ }^{2}$ Chemical and Clinical Pathology Department, Faculty of Medicine, Cairo University, Cairo, Egypt \\ ${ }^{3}$ Clinical Oncology Department, Faculty of Medicine, Cairo University, Cairo, Egypt \\ ${ }^{4}$ Palliative Medicine Unit, Kasr Al-Ainy Centre of Clinical Oncology \& Nuclear Medicine, Faculty of Medicine, Cairo \\ University, Cairo, Egypt
}

\author{
*Correspondence to \\ Soheir Shehata RezkAllah Rezk \\ Assistant professor, Department of \\ Basic Science, Faculty of Physical \\ Therapy, Cairo University. \\ 7 Ahmed Elzayyat st., Dokki, Giza, \\ Egypt. \\ Tel: 01224003374, 0224320635; \\ Email: \\ soheirsamaan@hotmail.com
}

Published online February 25, 2019

\begin{abstract}
Introduction: Oral mucositis (OM) is an unavoidable condition of the oral cavity that accompanies chemotherapy for various malignant cases. Chemotherapy-induced oral Mucositis (COM) is a frequent complication due to mucotoxic drugs and is known to deteriorate the general health significantly, while negatively affecting the quality of life (QOL). Studies have reported that low-level laser therapy (LLLT) promotes the tissue healing. The objective of the present study was to explore the efficacy of gallium-arsenide (GaAs) laser in treating COM and its impact on inflammatory cytokine levels in patients receiving chemotherapy for various malignancies.

Methods: A total of 80 patients with COM received LLLT 6 days/week. OM was graded according to the World Health Organization (WHO) grading scale. The outcome parameters were the serum levels of tumor necrosis factor-alpha (TNF- $\alpha$ ) and interleukin-6 (IL-6) measured before, during and after administration of LLLT.

Results: After LLLT, a significant decrease was found in the mean values of mucositis grade from $2.35 \pm 0.695$ to $1.13 \pm 0.333$ after $(P<0.001)$. A significant reduction in the level of TNF- $\alpha$ was found after LLLT among breast cancer patients $(P=0.0045)$, but not in head and neck cancer and lymphoma patients. A significant reduction was also found in IL-6 level after treatment among head and neck and breast cancer patients $(P=0.0307$ and 0.019 , respectively).

Conclusion: The use of GaAs LLLT in treating COM in patients with various malignancies is well tolerated by patients, it results in improvement of mucositis, however; mechanism of action does not seem to be completely linked to the change of pro or anti-inflammatory cytokines.

Keywords: Low-level laser therapy; Oral Mucositis; Cancer.
\end{abstract}

\section{Introduction}

Cancer patients who are receiving chemotherapy or radiotherapy are highly susceptible to have a very common complication which is oral mucositis (OM). Severe and wide spread ulcerated mucositis that happen in cancer patients compromise them both physiologically (by the tumor) and literally (by cancer therapy) to be placed at an increased risk of systemic infection and bleeding which can lead to impaired nutritional status and inadequate hydration. Consequently, the quality of life (QOL) of these patients will be greatly compromised, and can be associated with significant morbidity. ${ }^{1,2}$ The frequency and degree of chemotherapy-induced oral mucositis (COM) varies according to the used chemotherapeutic regimen. In breast cancer patients receiving taxol-anthracyclincyclophosphamide (TAC) combination chemotherapy, the average incidence of COM is high and approaches $65 \% .^{3}$ On the other hand, the average incidence is lower (approximately 15\%) in lung cancer patients treated with the platinum-gemcitabine combination. ${ }^{3}$ In addition to conventional chemotherapeutic agents, the development of newer anti-cancer agents, like epidermal growth factor receptor inhibitors and mammalian target of rapamycin inhibitors, increases the number of drugs that cause oral mucosal toxicities in the cancer care setting. ${ }^{4}$

The panoply of OM symptoms inevitably impacts a patients' QOL and their willingness to continue treatment and has a negative impact on them, ${ }^{5}$ this is due to severe pain and burning sensation which is a significant component of OM, dysphagia (difficulty in the feeding process) whatever food is liquid or solid, dysarthria (speech dysfunction due to impaired coordination of the

Please cite this article as follows: Rezk-Allah SS, Elshafi HMA, Farid RJ, Hassan MAE, Alsirafy SA. Effect of low-level laser therapy in treatment of chemotherapy induced oral mucositis. J Lasers Med Sci. 2019;10(2):125-130. doi:10.15171/jlms.2019.20. 
muscles responsible for word articulation), odynophagia (difficulty of swallowing due to pain or burning sensation), also as oral lesions provide good environment for opportunistic infections that can be life-threatening infections (septicemia in neutropenic cases), it can also have a negative impact in delaying administration of radiation therapy and or chemotherapy, dose reduction of the chemotherapeutic drugs. ${ }^{6}$

The negative impact of $\mathrm{OM}$ on cancer patients is not limited to pain and physical consequences and extends to other aspects of QOL, like the psychological and social aspects. ${ }^{7,8}$ Furthermore, OM may result in a significant economic cost. ${ }^{7}$

Amelioration of mucositis can greatly affect the feeding capacity of patients, through pain relief and improving symptoms associated with chewing and swallowing. It also allows lowering the frequency and cost of hospitalization and palliative care. ${ }^{9}$ The management of $\mathrm{OM}$ is necessary to improve the QOL of cancer patients and to optimize their treatment outcome by preventing treatment interruptions. ${ }^{2}$ Many approaches to manage OM in cancer patients have been evaluated in clinical studies; like oral hygiene protocols, cryotherapy, benzydamine mouthwash and low-level laser therapy (LLLT). ${ }^{10,11}$

LLLT was proved to have positive effects on the patient's QOL in cancer patients suffering from an oral complication during oncotherapy treatment, ${ }^{12}$ and it was stated that laser therapy should become part of nutritional interventions in oncological patients affected by OM. ${ }^{9}$

LLLT has several applications in the supportive care of patients with cancer. For example, LLLT was found to be effective in the management of OM, lymphedema, and chemotherapy-induced peripheral neuropathy. ${ }^{13}$

A systemic literature review was done ${ }^{14}$ to assess the effectiveness of LLLT as a preventive and therapeutic modality of OM in cancer patients 27 articles were assessed, 3 of them were animal studies and 24 were human studies. The results showed that LLLT is a noninvasive modality for prevention and management of $\mathrm{OM}$ with some advantages like analgesic effect, inflammation reduction and atraumatic repeated use.

Another systemic review and meta-analysis were conducted in which 12 studies were included, 7 studies showed that application LLLT in patients put on oncotherapy is nearly nine times more effective to prevent OM grade $>3$ than in patients who did not received laser therapy. These data dedicate that LLLT is considered a prophylactic treatment for patients with OM (grade $>3$ ).$^{15}$

The evidence that LLLT has a role in treating OM in cancer patients justified its inclusion in international mucositis management guidelines. The updated Multinational Association of Supportive Care in Cancer and International Society of Oral Oncology (MASCC/ ISOO) Clinical Practice Guidelines for OM included LLLT $^{10}$ The MASCC/ISOO guidelines included one recommendation and one suggestion for using LLLT. The recommendation is to use LLLT to prevent OM in patients put on chemotherapy of high doses and being prepared for hematopoietic stem cell transplantation. In addition, the suggestion is to use it to avoid the development of OM in head and neck cancer patients receiving radiotherapy without concomitant chemotherapy. ${ }^{10}$ The available evidence as of 2014 was not strong enough to recommend/ suggest the use of LLLT for OM in cancer patients in other clinical settings.

Although there is good evidence supporting the application of LLLT in patients put on high-dose chemotherapy, there is still a need to investigate the optimal LLLT characteristics and its feasibility in COM. ${ }^{16}$

The current study was planned to explore the effectiveness of gallium-arsenide (GaAs) laser therapy in the healing of COM and its relation to the level of inflammatory cytokines; tumor necrosis factor-alpha (TNF- $\alpha$ ) as well as interleukin-6 (IL-6) in cancer patients.

\section{Methods}

Trial Design and Participants

This study is one group pretest-posttest design (quasiexperimental design). It was conducted at Kasr ElAini Center of Clinical Oncology and Nuclear Medicine, Cairo, Egypt in the period from January to September 2017. Eighty patients with COM were selected from the out and inpatient clinic.

The study included 80 patients with the following eligibility criteria: adult patients ( $>18$ years old), pathological diagnosis of cancer (breast, head and neck or non-Hodgkin's lymphoma), recent exposure to inductive chemotherapy phase within 2 weeks and OM. Only those head and neck cancer patients were included if they did not receive radiotherapy. Chemotherapy administered using standard protocols and included at least one of these agents: cisplatin, 5-fluorouracil, taxanes, vincristine, anthracyclines, gemcitabine or methotrexate; and is planned to be administered for 4 to 8 cycles with 2 to 3 weeks intervals. A consent form was signed by all eligible patients.

Patients were excluded under the following conditions: double malignancy or metastases of unknown origin, chemotherapy phases other than inductive, reduced mouth opening $\left(<1 \mathrm{~cm}^{2}\right)$, severe uncontrolled collagen vascular disease or pregnancy, current smokers.

\section{Assessment of Oral Mucositis}

The severity of OM was rated according to the World Health Organization (WHO) grading of mucositis scale ${ }^{17}$ as follows:

- Score 0: no signs or symptoms.

- Score 1: Oral soreness and erythema.

- Score 2: Oral erythema and ulcers, both solid and liquid diets tolerated.

- Score 3: Oral ulcers, liquid diet only.

- Score 4: Oral alimentation impossible. 
Measurement of Pro-inflammatory Cytokines

Serum TNF- $\alpha$ and IL-6 were estimated using enzymelinked immunosorbent assay (ELISA). The serum level of both cytokines was measured 4 times during the whole treatment period of the patient (one time before treatment, at the second and fourth weeks of treatment, and at the end of treatment).

\section{Laser Therapy}

Laser therapy was performed using laser Phyaction CL904 device (Uniphy technology, Belgium) with a small hand held probe class $3 \mathrm{~B}$. The device produces pulsed infrared GaAs laser with wavelength of $904 \mathrm{~nm}$, peak power of $25 \mathrm{~W}$, pulse duration of $200 \mathrm{~ns}$, and energy density of $1 \mathrm{~J} / \mathrm{cm}^{2} .{ }^{17}$

Calibration of the laser Phyaction CL device was performed according to the specifications outlined by the manufacturer's service manual before each session.

Treatment with laser therapy was done 6 days/week from the start of OM till the end of chemotherapy. Laser therapy was applied on each point for 1 minute with energy density of $3 \mathrm{~J} / \mathrm{cm}^{2}$.

All patients received routine oral care, local antifungal and analgesics (acetaminophen) and nutritional support during the laser treatment. They were advised to follow a daily mouth hygienic care. Photographic picture to the oral cavity was taken before and after laser therapy.

\section{Statistical Methods}

Data were represented as mean \pm standard deviation or as percentages when appropriate. Paired sample $t$ test was used to test the significant difference in the mucositis grade and the level of TNF- $\alpha$ and IL- 6 before and after LLLT therapy.

\section{Results}

The study included 80 cancer patients with COM. Their characteristics are shown in Table 1. Almost half of the patients had grade 3 OM before starting laser therapy. Chi-square test was used to test the significance of the difference in mucositis grades before and after treatment. The level of significance was set at a $P$ value $<0.05$.

The average grade of mucositis decreased significantly from $2.35 \pm 0.695$ before LLLT therapy to $1.13 \pm 0.333$ after $(P<0.001)$ as shown in Figure 1 . The percentage of improvement was $87.5 \%$.

The distribution of mucositis grades before and after laser therapy is shown in Table 2. A significant decrease in the severity of mucositis was found after therapy. For the whole group of patients, the change in TNF- $\alpha$ level after treatment was not significant (Table 3). However, a significant decrease was found in TNF- $\alpha$ after treatment in breast cancer patients but not head and neck and lymphoma patients.

For the whole group of patients, the change in IL-6 level after LLLT was not significant (Table 4). A significant
Table 1. Patients' Characteristics

\begin{tabular}{lcc}
\hline Character & Value & Percent \\
\hline Age $(y)$ & & \\
Median (range) & 55.2 & $(40-65)$ \\
$>50$ & 46 & $57.5 \%$ \\
$<50$ & 34 & $42.5 \%$ \\
Sex & & \\
Female & 43 & $53.8 \%$ \\
Male & 37 & $46.3 \%$ \\
Smoking (before cancer) & & \\
Yes & 31 & $38.8 \%$ \\
No & 49 & $61.3 \%$ \\
Primary cancer & & \\
\hline Head and neck cancer & 33 & $41.3 \%$ \\
Non-Hodgkin's lymphoma & 28 & $35 \%$ \\
Breast cancer & 19 & $36.3 \%$ \\
\hline Pre-treatment mucositis grade & & \\
\hline 1 & 10 & $12.5 \%$ \\
2 & 32 & $40 \%$ \\
\hline Mean of WHO assessment scale pre-treatment \pm SD & $2.35 \pm 0.695$ \\
\hline
\end{tabular}

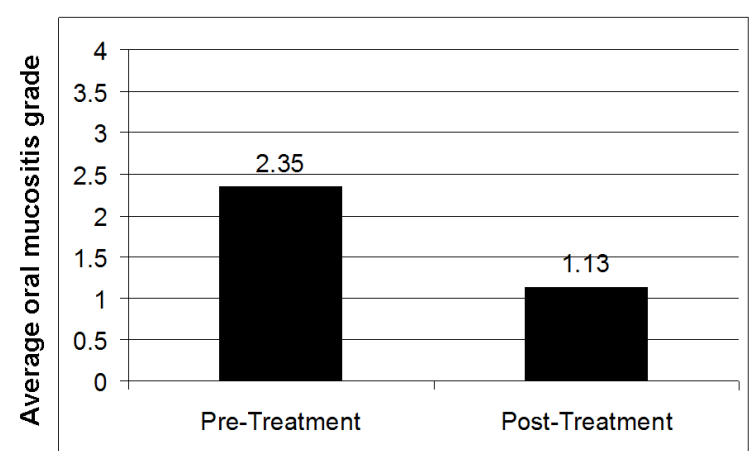

Figure 1. Grade of OM Before and After GaAs LLLT.

Table 2. Distribution of Mucositis Grades Before and After Treatment

\begin{tabular}{lccccc}
\hline \multirow{2}{*}{$\begin{array}{l}\text { Mucositis } \\
\text { Grade }\end{array}$} & \multicolumn{2}{c}{ Pre-treatment } & \multicolumn{2}{c}{ Post-treatment } & \multirow{2}{*}{$\boldsymbol{P}$ Value } \\
\cline { 2 - 5 } & No. & $\%$ & No. & $\%$ & \\
\hline 1 & 10 & 12.5 & 70 & 87.5 & 0.001 \\
2 & 32 & 40 & 10 & 12.5 & \\
3 & 38 & 47.5 & 0 & 0 & \\
\hline
\end{tabular}

decrease in IL-6 level was found after LLLT among head \& neck and breast cancer patients. On the other hand, a significant increase in its level was found among lymphoma patients after laser therapy.

There was no significant correlation between the posttreatment levels of TNF- $\alpha$ and IL- 6 in patients with head and neck cancer $(\mathrm{r}=-0.9159, P=0.263)$, lymphoma patients $(\mathrm{r}=0.6835, P=0.5209)$, nor breast cancer patients $(\mathrm{r}=0.8431, P=0.1569)$. 
Table 3. TNF-a Level Before and After Treatment

\begin{tabular}{lccc}
\hline Variable & Pre-treatment & Post-treatment & t-test \\
\cline { 2 - 3 } & Mean $( \pm$ SD) & Mean $( \pm$ SD) & 0.3964 \\
All patients & $92.31 \pm 17.06$ & $104.9 \pm 26.91$ & 0.6965 \\
Primary cancer & & & 1.046 \\
$\quad$ Head \& neck cancer & $92.87 \pm 41.06$ & $153.2 \pm 40.47$ & 1.338 \\
$\quad$ Lymphoma & $105.3 \pm 45.87$ & $173.9 \pm 22.83$ & 0.354 \\
Breast cancer & $82.13 \pm 14.54$ & $17.05 \pm 2.267$ & 0.2518 \\
\hline
\end{tabular}

Table 4. IL-6 Level Before and After Treatment

\begin{tabular}{lccc}
\hline Variable & Pre-treatment & Post-treatment & \multirow{2}{*}{ t-test } \\
\cline { 2 - 3 } & Mean $( \pm$ SD) & Mean $( \pm$ SD) & 1.818 \\
All patients & $99.23 \pm 11.19$ & $59.93 \pm 18.50$ & 0.0827 \\
Primary cancer & & & \\
$\quad$ Head \& neck cancer & $94.10 \pm 27.17$ & $17.30 \pm 2.676$ & 2.813 \\
$\quad$ Lymphoma & $104.1 \pm 12.23$ & $145.4 \pm 9.817$ & 2.634 \\
Breast cancer & $99.48 \pm 21.74$ & $26.93 \pm 6.813$ & 0.0307 \\
\hline
\end{tabular}

\section{Discussion}

This work was conducted to explore the effectiveness of GaAs LLLT in treating COM in patients with three types of cancer, breast, head and neck cancers and nonHodgkin's lymphoma. Overall, the results of the current study showed that GaAs LLLT is effective in treating COM. Our results confirm the accumulating evidence that LLLT is a therapeutic modality that is effective in treating $\mathrm{OM}$ in cancer patients. ${ }^{2,10}$

The strong evidence that LLLT has a role in the management of OM resulting from anti-cancer therapy has led to its inclusion in international mucositis management guidelines. The MASCC/ISOO guidelines recommended LLLT to prevent OM in patients put on high-dose chemotherapy and are being prepared for hematopoietic stem cell transplantation and suggested to use it to prevent $\mathrm{OM}$ in head and neck cancer patients receiving radiotherapy without concomitant chemotherapy. ${ }^{10}$ Because there is no sufficient evidence, the MASCC/ISOO guidelines did not recommend LLLT for the management of COM in patients receiving conventional chemotherapy protocols. The current study investigated LLLT for COM in cancer patients receiving conventional chemotherapy protocols and the results concur with those of some other studies, ${ }^{19-21}$ we found a significant decrease in the mean scores of
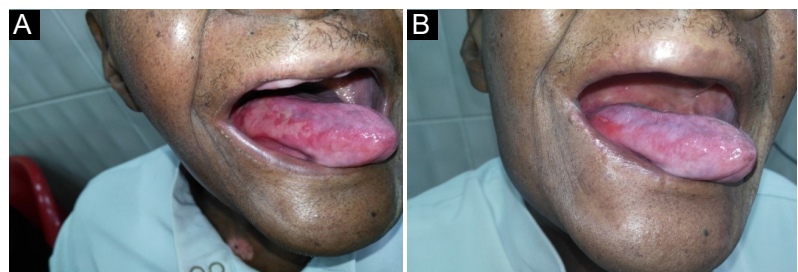

Figure 2. Male Patient With Oral Mucositis (A) before and (B) GaAs LLLT. mucositis after LLLT. Grade 3 mucositis decrease from $48 \%$ before treatment with LLLT to $0 \%$ after treatment. In their randomized controlled trial, they found that prophylactic LLLT reduced significantly the grade of COM, xerostomia, and pain in a group of adult cancer patients receiving chemotherapy. ${ }^{19}$

However, the ability of LLLT to reduce the grade of COM is inconsistent among studies. In a randomized controlled trial conducted, ${ }^{20}$ the time to healing of mucositis and pain were significantly less in patients treated with laser. However, there was no significant difference in the grade of COM. Similarly, in another randomized controlled trial conducted, ${ }^{21}$ LLLT reduced pain significantly, but not the grade of COM in young cancer patients.

The results of the current study and those of other studies highlight the need for further clinical trials to evaluate the role of LLLT in COM among patients receiving conventional chemotherapeutic protocols.

Cytokines play a major role in the pathogenesis of anti-cancer therapy induced $\mathrm{OM}$ and understanding their role may be helpful in developing management strategies. ${ }^{10,22,23}$ Among these, are the pro-inflammatory cytokines IL- 6 and TNF- $\alpha$ which were studied and conducted in more than one study, ${ }^{22-24}$ in which they examined the impact of LLLT on inflammatory cytokines during COM among patients undergoing hematopoietic stem cell transplantation. They found a significant difference in plasma level of IL-10 only. No significant difference was found in other mediators, TNF- $\alpha$, IL- 6 , IL- $1 \beta$, TGF- $\beta$. They concluded that the mechanism by which LLLT reduces COM may not be completely linked to the modulation of inflammatory cytokines levels. ${ }^{24}$ Similar to their study, ${ }^{24}$ in our study, TNF- $a$ did not differ significantly after treating COM in head and neck cancer and lymphoma patients. However, there was significant 
decrease in TNF- $\alpha$ in breast cancer patients. Unlike their study, ${ }^{25}$ a significant change was found in IL-6 level. However, the change was differential according to the tumor type. Serum level of IL-6 decreased in patients with head and neck and breast cancer, and increased in patients with lymphoma after laser therapy, this may be due to the role of IL-6 expression in cellular sensitivity to LLLT. ${ }^{26}$

The findings of the current study and those of other studies show that the relation between the healing effect of LLLT and modulation of inflammatory cytokines in COM is not completely understood and needs further investigation. Since one of the major limitations of present study is the lack of a control group; a randomized controlled trial would yield better evidence for clinical application.

There are other areas that need exploration, like the combination of LLLT with other mucositis treatment modalities. The addition of cryotherapy to laser therapy resulted in significantly more reduction in the severity and duration of melphalan-induced $\mathrm{OM}$ when compared to laser therapy alone. ${ }^{27}$ In a recent RCT, the combination of LLLT and photochemotherapy resulted in significantly more reduction in the severity of COM when compared to LLLT alone in young cancer patients. ${ }^{28}$

\section{Conclusion}

Regarding the findings of the current study, the use of GaAs LLLT in the management of COM in cancer patients receiving conventional chemotherapy is well tolerated and results in the improvement of OM. It is able to reduce the proinflammatory cytokines; IL6 and TNF- $\alpha$ in head and neck cancer and breast cancer patients. Therefore it may be useful to improve the symptoms of chemotherapy- induced OM.

\section{Funding}

The author of this article did not have any sort of funding for the preparation of this work.

\section{Conflict of Interests}

Authors of this paper have no conflict of interest.

\section{Ethical Considerations}

The study was approved by the ethical committee of the Faculty of Physical Therapy, Cairo University. All participants signed an informed consent form.

\section{Acknowledgments}

The authors are grateful to the volunteers and participants involved in this work.

\section{References}

1. Bensadoun R, Nair R, Pine B, Caillot E, Berger A, Jarde P. Laser parameters drawn from meta-analysis on the efficacy of LLLT in oral mucositis. Support Care Cancer.
2012; 20(1):1-283. doi:10.1007/s00520-011-1202-0

2. Campos MI, Campos CN, Aarestrup FM, Aarestrup BJ. Oral mucositis in cancer treatment:Natural history, prevention and treatment. Mol Clin Oncol. 2014;2(3):33740. doi: $10.3892 /$ mco. 2014.253

3. Jones JA, Avritscher EB, Cooksley CD, Michelet M, Bekele BN, Elting LS. Epidemiology of treatment-associated mucosal injury after treatment with newer regimens for lymphoma, breast, lung, or colorectal cancer. Support Care Cancer. 2006;14(6):505-15. doi:10.1007/s00520-006-00554

4. Vigarios E, Epstein JB, Sibaud V. Oral mucosal changes induced by anticancer targeted therapies and immune checkpoint inhibitors. Support Care Cancer. 2017;25(5):1713-9. doi:10.1007/s00520-017-3629-4

5. Bonomi M. and Batt K. Supportive management of mucositis and metabolic derangements in head and neck cancer patients. Cancers (Basel). 2015;7:1743-57. doi: $10.3390 /$ cancers7030862

6. Gholizadeh N, Sheykhbahaei N, Sadrzadeh-Afshar M. New treatment approaches of oral mucositis: a review of literature. Adv Hum Biol. 2016;6:66-72. doi:10.4103/23218568.190319

7. Lalla RV, Saunders DP, Peterson DE. Chemotherapy or radiation-induced oral mucositis. Dent Clin North Am. 2014;58(2):341-9. doi:10.1016/j.cden.2013.12.005

8. Barkokebas A, Silva IH, de Andrade SC, et al. Impact of oral mucositis on oral-health-related quality of life of patients diagnosed with cancer. J Oral Pathol Med. 2015;44(9):74651. doi:10.1111/jop.12282

9. Gobbo M, Ottaviani G, Perinetti G, et al. Evaluation of nutritional status in head and neck radio-treated patients affected by oral mucositis: efficacy of class IV laser therapy. Support Care Cancer. 2014;22(7):1851-1856. doi: 10.1007/ s00520-014-2155-x.

10. Lalla RV, Bowen J, Barasch A, et al. MASCC/ISOO clinical practice guidelines for the management of mucositis secondary to cancer therapy. Cancer. 2014;120(10):145361. doi:10.1002/cncr.28592

11. Chaveli-López B, Bagán-Sebastián JV. Treatment of oral mucositis due to chemotherapy. J Clin Exp Dent. 2016;8(2):e201-e209. doi:10.4317/jced.52917

12. Antunes H, Herchenhorn D, Small I, et al. Phase III trial of low-level laser therapy to prevent oral mucositis in head and neck cancer patients treated with concurrent chemoradiation. Radiother Oncol. 2013;109:297-302.

13. Robijns J, Censabella S, Bulens P, Maes A, Mebis J. The use of low-level light therapy in supportive care for patients with breast cancer: review of the literature. Lasers Med Sci. 2017;32(1):229-42. doi:10.1007/s10103-016-2056-y

14. Fekrazad R, Chiniforush N. Oral mucositis prevention and management by therapeutic laser in head and neck cancers. J Lasers Med Sci. 2014;5(1):1-7.

15. Figueiredo A, Lins L, Cattony A, Falcão A. Laser therapy in the control of oral mucositis:a meta-analysis. Rev Assoc Med Bras (1992). 2013;59(5):467-474. doi:10.1016/j. ramb.2013.08.003

16. Oberoi S, Zamperlini-Netto G, Beyene J, Treister NS, Sung L. Effect of prophylactic low level laser therapy on oral mucositis: a systematic review and meta-analysis. PLoS One. 2014;9(9):e107418. doi:10.1371/journal. 


\section{pone. 0107418}

17. World Health Organization. Handbook for reporting results of cancer treatment. Genava: World Health Organization; 1979:15-22.

18. Lanz B, Kostamovaara JT. Current pulse investigation toward optimal pumping of a gain-switched asymmetric waveguide laser diode. Proceedings of SPIE Laser Technology for Defense and Security X Conference (DSS); Baltimore, USA; 5- 9 May 2014: 90810W 1-10.

19. Arbabi-Kalati F, Arbabi-Kalati F, Moridi T. Evaluation of the effect of low level laser on prevention of chemotherapyinduced mucositis. Acta Med Iran. 2013;51(3):157-162.

20. Ahmed KM, Hussein SA, Noori AJ, Abdulateef SN, Abdulla BK. Evaluation of low level laser therapy in the management of chemotherapy-induced oral mucositis in pediatric and young cancer patients: a randomized clinical trial. European Scientific Journal. 2015;11(27):209-222.

21. Amadori F, Bardellini E, Conti G, Pedrini N, Schumacher RF, Majorana A. Low-level laser therapy for treatment of chemotherapy-induced oral mucositis in childhood: a randomized double-blind controlled study. Lasers Med Sci. 2016;31(6):1231-1236

22. Ahad A, Tasneem S, Lamba AK, Khan S. Healing of selfinflicted thermal injury of palatal mucosa by low level laser therapy. Spec Care Dentist. 2017;37(6):314-317. doi: $10.1111 / \mathrm{scd} .12256$

23. Logan RM, Stringer AM, Bowen JM, et al. The role of pro-inflammatory cytokines in cancer treatment-induced alimentary tract mucositis: pathobiology, animal models and cytotoxic drugs. Cancer Treat Rev. 2007;33(5):448460. doi:10.1016/j.ctrv.2007.03.001

24. Raber-Durlacher JE, von Bültzingslöwen I, Logan RM, et al. Systematic review of cytokines and growth factors for the management of oral mucositis in cancer patients. Support Care Cancer. 2013;21(1):343-355. doi:10.1007/ s00520-012-1594-5

25. Silva GB, Sacono NT, Othon-Leite AF, et al. Effect of lowlevel laser therapy on inflammatory mediator release during chemotherapy-induced oral mucositis: a randomized preliminary study. Lasers Med Sci. 2015;30(1):117-126. doi:10.1007/s10103-014-1624-2

26. Usuda J, Okunaka T, Furukawa K, et al. Increased cytotoxic effects of photodynamic therapy in IL- 6 gene transfected cells via enhanced apoptosis. IJC. 2001;39(4):475-80.

27. de Paula Eduardo F, Bezinelli LM, da Graça Lopes RM, Nascimento Sobrinho JJ, Hamerschlak N, Correa L. Efficacy of cryotherapy associated with laser therapy for decreasing severity of melphalan-induced oral mucositis during hematological stem-cell transplantation:a prospective clinical study. Hematol Oncol. 2015;33(3):152158. doi:10.1002/hon.2133

28. Medeiros-Filho JB, Maia Filho EM, Ferreira MC. Laser and photochemotherapy for the treatment of oral mucositis in young patients: randomized clinical trial. Photodiagnosis Photodyn Ther. 2017;18:39-45. doi:10.1016/j. pdpdt.2017.01.004 